Ecología

\title{
Dujardinascaris helicina (Nematoda: Ascarididae) parásito de Crocodylus acutus (Reptilia: Crocodylidae) en Puerto Vallarta, Jalisco, México
}

\author{
Dujardinascaris helicina (Nematoda: Ascarididae), parasite of Crocodylus acutus \\ (Reptilia: Crocodylidae) in Puerto Vallarta, Jalisco, Mexico \\ Jonathan Nácar-Muñoz $^{\text {a,* }}$, Virginia León-Règagnon ${ }^{\mathrm{b}}$, Juan L. Cifuentes-Lemus ${ }^{\mathrm{a}}$, \\ Helios Hernández-Hurtado ${ }^{a}$, Pablo Hernández-Hurtado ${ }^{a}$ y Rosa M. Chávez-Dagostino a \\ a Departamento de Ciencias Biológicas, Centro Universitario de la Costa, Universidad de Guadalajara, Av. Universidad 203, Delegacion Ixtapa, 48280, \\ Puerto Vallarta, Jalisco, México \\ b Estación de Biología Chamela, Sede Colima, Instituto de Biología, Universidad Nacional Autónoma de México, 48980, San Patricio, Jalisco, México
}

Recibido el 22 de septiembre de 2015; aceptado el 19 de mayo de 2016

Disponible en Internet el 21 de agosto de 2016

\section{Resumen}

El cocodrilo de río (Crocodylus acutus, Cuvier, 1807) es una especie protegida en México. En este trabajo se han estudiado los contenidos obtenidos por medio de lavados gástricos a 53 C. acutus, en las áreas del estero el Salado, estero Boca Negra-Boca de Tomates y en la UMA Reptilario Cipactli, ubicadas en Puerto Vallarta, Jalisco, México, con el fin de conocer la helmintofauna de la especie. Se identificó solamente al nemátodo, Dujardinascaris helicina (Molin, 1860). La mayor prevalencia fue del 74\% y la abundancia 3.17 en el área del estero Boca Negra-Boca de Tomates; y la menor prevalencia fue del $12 \%$, con una abundancia de 0.76 en los ejemplares en la UMA Reptilario Cipactli. Suponemos que esta diferencia se debe principalmente al tipo de alimento suministrado a los cocodrilos en cautiverio. En esta investigación se registra por primera vez a D. helicina como parásito de $C$. acutus en la región.

Derechos Reservados @ 2016 Universidad Nacional Autónoma de México, Instituto de Biología. Este es un artículo de acceso abierto distribuido bajo los términos de la Licencia Creative Commons CC BY-NC-ND 4.0.

Palabras clave: Nemátodo; Prevalencia

\section{Abstract}

The river crocodile (Crocodylus acutus, Cuvier, 1807) is a protected species in Mexico. In this work the stomach contents obtained through gastric lavage of 53 C. acutus from the estuarine systems "El Salado", "Boca Negra-Boca de Tomates" and from the "UMA (Unidad de Manejo y Conservación de Vida Silvestre" for its meaning in Spanish) Reptilario Cipactli" located in Puerto Vallarta, Jalisco, Mexico were collected to study the helminth fauna of the species. Only Dujardinascaris helicina (Molin, 1860) was identified. The highest prevalence occurred in "Boca Negra-Boca de Tomates" with 74\% and an abundance of 3.17, and the lowest in "UMA Reptilario Cipactli" with $12 \%$ and an abundance of 0.76 . The dissimilarity is assumed to be caused primarily by the different feeding habits between wildlife and captivity raised crocodiles. During this investigation D. helicina is registered for the first time as a parasite for C. acutus in the region.

All Rights Reserved (C) 2016 Universidad Nacional Autónoma de México, Instituto de Biología. This is an open access item distributed under the Creative Commons CC License BY-NC-ND 4.0.

Keywords: Nematode; Prevalence

\footnotetext{
* Autor para correspondencia.

Correo electrónico: onisalit@ gmail.com (J. Nácar-Muñoz).

La revisión por pares es responsabilidad de la Universidad Nacional Autónoma de México.
} 


\section{Introducción}

En México, la familia Crocodylidae está representada por 2 especies, C. moreletii y C. acutus. Ésta última, llamada cocodrilo de río, se distribuye a lo largo de las costas del Atlántico y Pacífico de México, sur de Florida, América Central, América del Sur y en diversas islas del Caribe (Thorbjarnarson, 1989). Además, está protegida por la Norma Oficial Mexicana NOM059-SEMARNAT-2010 y en el apéndice 1 de la Convención sobre el Comercio Internacional de Especies Amenazadas de Fauna y Flora Silvestres (CITES, 2007).

Por otro lado, los estudios helmintológicos realizados en cocodrilos de México son escasos. La especie de helminto encontrado en cocodrilos pertenece a la familia Ascarididae, la más frecuente de nemátodos en cocodrilos (Brooks y O'Grady, 1989; Goldberg, Bursey y Aquino-Shuster, 1991).

El género Dujardinascaris se ha registrado en peces y reptiles (Sprent, 1977). Es un género cosmopolita con 18 especies descritas hasta ahora y casi todas parásitas de Crocodylia (Baker, 1987; Goldberg et al., 1991; Sprent, 1977; Sprent, McKeown y Cremin, 1998). Dujardinascaris helicina fue descrita originalmente por Molin (1860) a partir de gusanos recolectados en ejemplares de $C$. acutus depositados en un museo. Se trata de un nemátodo común en cocodrilos de agua dulce y agua salada: $C$. acutus, C. moreletii y C. rhombifer (Groschaft y Baruš, 1970; Moravec, 2001). En México, esta especie fue registrada con alta prevalencia y abundancia en el tracto digestivo de $C$. moreletii de Yucatán (Moravec, 2001) y en un ejemplar de C. moreletii en Quintana Roo (Villegas y Gonzáles-Solís, 2009), las investigaciones se han centrado principalmente en $C$. moreletii (Álvarez-del Toro, 1974; Moravec, 2001; Moravec y VargasVázquez, 1998; Sprent, 1977). Los únicos estudios que en $C$. acutus se han realizado en el sur del país, en Quintana Roo (Villegas y González-Solís, 2009) y Río Verde, Oaxaca (Peláez y Cruz, 1953). En este trabajo se registra por primera vez a D. helicina como parásito de C. acutus, en la costa de Puerto Vallarta, México.

\section{Materiales y métodos}

De junio a noviembre de 2011 se realizaron lavados gástricos a 36 ejemplares de $C$. acutus que habitan 2 diferentes zonas de Puerto Vallarta: estero El Salado $\left(20^{\circ} 39^{\prime} 46^{\prime \prime} \mathrm{N}\right.$, $105^{\circ} 14$ '34' O) y estero Boca Negra-Boca de Tomates (2040'7' $\left.\mathrm{N}, 105^{\circ} 16^{\prime} 11.94^{\prime \prime} \mathrm{O}\right)$ y a 17 que viven en cautiverio en la UMA reptilario Cipactli $\left(20^{\circ} 42^{\prime} 16^{\prime}\right.$ ' N, 105 $13^{\prime} 16^{\prime \prime} \mathrm{O}$; fig. 1). Los cocodrilos se capturaron, dependiendo del tamaño del ejemplar, mediante el uso de embarcaciones pequeñas y cuerdas, pértigas

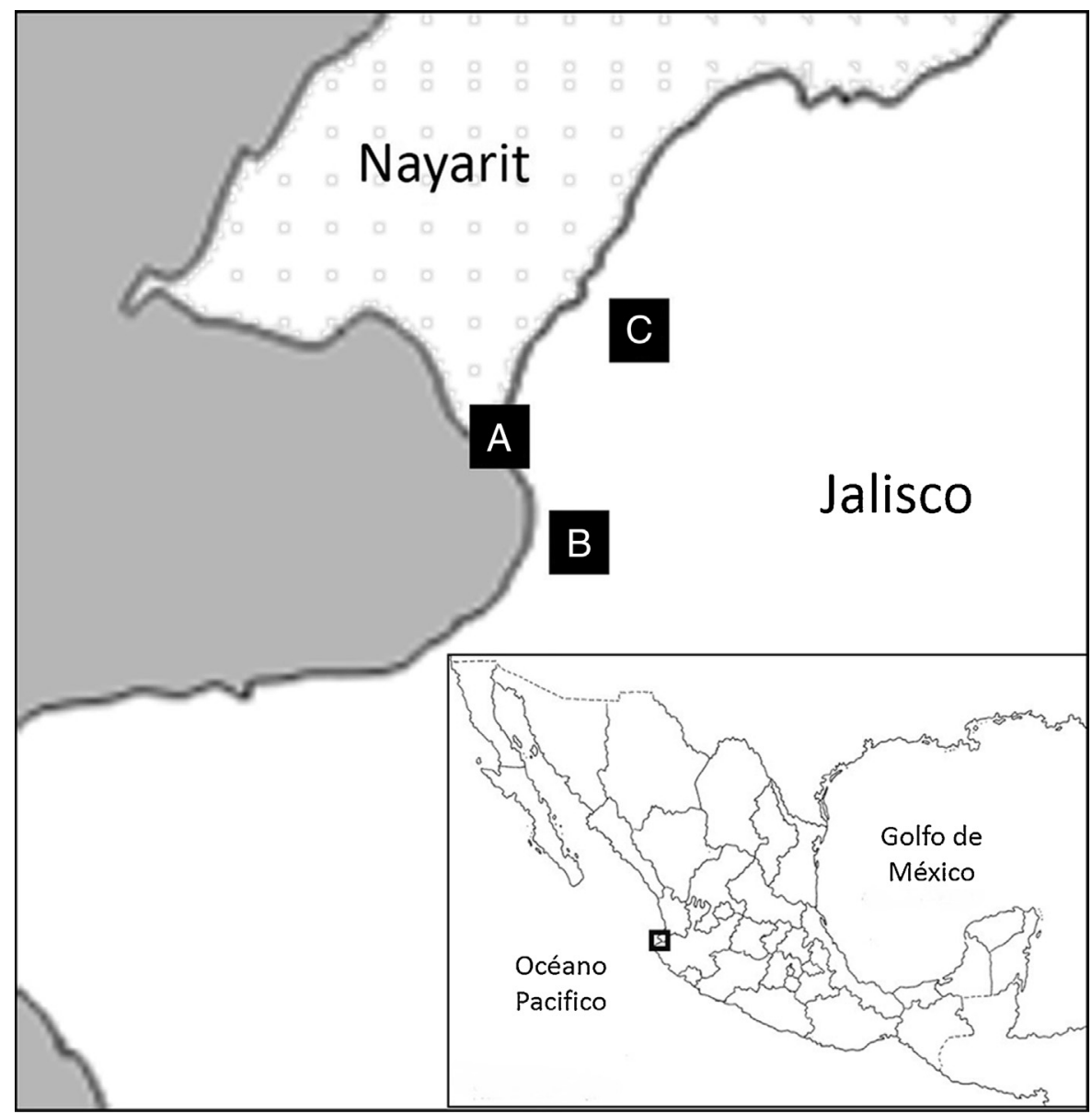

Figura 1. Áreas de estudio donde se capturó Crocodylus acutus: A) estero Boca Negra-Boca de Tomates; B) estero El Salado; C) UMA reptilario Cipactli. 
o las manos. La extracción del contenido estomacal se realizó con el método de Ayarzagüena (1983) y la maniobra de Heimlich (1975), que consisten en mantener las mandíbulas del cocodrilo abiertas para introducir una sonda hasta el estómago. Una vez que la sonda llega hasta allí, se inyecta agua hasta que el estómago quede completamente lleno; se masajea el abdomen y al mismo tiempo se inclina al cocodrilo con la cabeza hacia abajo, para que por gravedad el contenido estomacal salga por la sonda y se coloca en frascos. Los nemátodos obtenidos fueron fijados y conservados en etanol al $70 \%$ y posteriormente, para su identificación morfológica, fueron aclarados en glicerol. Se efectuó su descripción morfológica y métrica para su posterior identificación. Las medidas se expresan en milímetros $(\mathrm{mm})$ y en promedio, entre paréntesis la desviación estándar. Se calcularon los indicadores de infección parasitaria propuestos por Bush, Lafferaty, Lotz y Shostak (1997). Los ejemplares identificados fueron depositados en la Colección Nacional de Helmintos de la Universidad Nacional Autónoma de México, con número de catálogo previo 8839 y en la UMA Reptilario Cipactli del Centro Universitario de la Costa de la Universidad de Guadalajara.

\section{Resultados}

En el contenido gástrico de 21 de los 36 cocodrilos de vida libre examinados y en 3 de los 17 en cautiverio se localizaron nemátodos. Los nemátodos adultos aislados fueron identificados como D. helicina (Molin, 1860). Los nemátodos son de color rosado, cutícula con estrías transversales; el extremo posterior del cuerpo enrollado; la boca está rodeada por 3 labios y 3 interlabios, los huevos son esféricos (fig. 2). Las mayores prevalencias y abundancias medias de infección de $D$. helicina en $C$. acutus se observaron en estero Boca Negra-Boca de Tomates y estero el Salado, y los menores valores en cocodrilos de la UMA Reptilario Cipactli (tabla 1).

Machos (17 ejemplares): largo total 11.86 (1.27), ancho máximo 0.32 (0.03). Largo labio 0.08 (0.008). Interlabio 0.05 (0.008). Esófago; 1.86 largo (0.14). Ciego 1.14 (0.02). Distancia extremo anterior: anillo nervioso $0.37(0.03)$, poro excretor 0.40 (0.03). Largo espículas 4.14 (0.05), ancho $0.02(0.006)$. Gubernáculo 0.12 (0.01). Cola 0.17 (0.01). Hembras (20 ejemplares): largo total 14.85 (0.75), ancho máximo 0.47 (0.04).

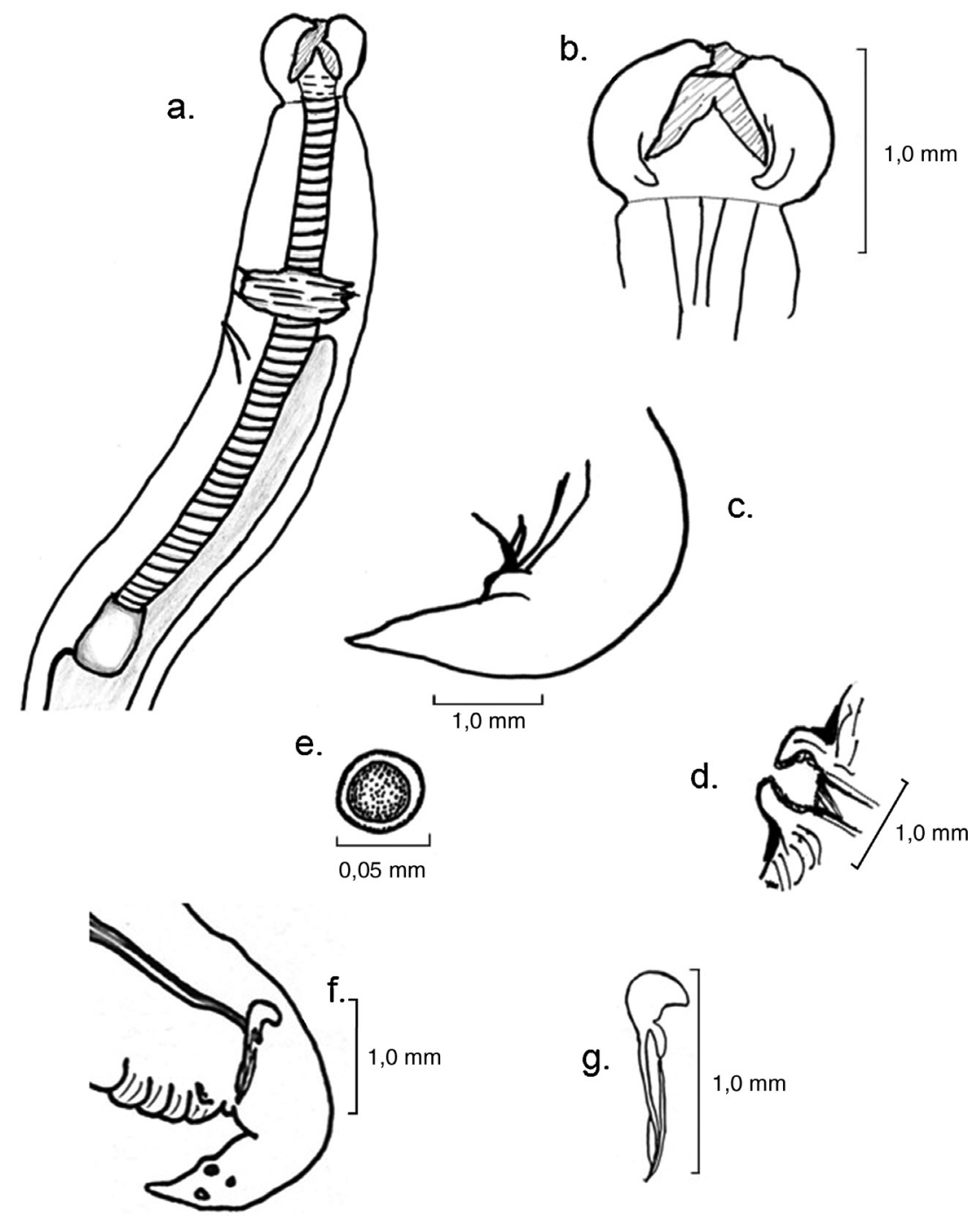

Figura 2. Dujardinascaris helicina: a) extremo anterior de la hembra; vista lateral; b) vista lateral de la cavidad oral; c) extremo posterior de la hembra; d) vulva; e) huevo; f) macho, extremo posterior; g) gubernáculo. 
Tabla 1

Indicadores de infección parasitaria de Dujardinascaris helicina en Crocodylus acutus según localidad de muestreo.

\begin{tabular}{lcccc}
\hline Localidad & \multicolumn{2}{c}{ Intensidad } & $\begin{array}{c}\text { Abundancia } \\
\text { media }\end{array}$ \\
\cline { 2 - 5 } & Machos & Hembras & $13 / 9(69.23)$ \\
\hline Estero El Salado $(\mathrm{n}=51)$ & 9 & 42 & $23 / 17(73.91)$ & 3.92 \\
Estero Boca Negra-Boca de Tomates $(\mathrm{n}=96)$ & 11 & 85 & 4.17 \\
UMA reptilario Cipactli $(\mathrm{n}=13)$ & 1 & 12 & $17 / 3(11.76)$ \\
\hline
\end{tabular}

$\mathrm{n}$ : número de nemátodos encontrados.

Largo labio 0.07 (0.004). Interlabio 0.05 (0.006). Largo esófago 2.36 (0.08). Ciego 1.99 (0.23). Distancia extremo anterior: anillo nervioso $0.41(0.01)$, poro excretor $0.4(0.01)$. Distancia extremo posterior-vulva $6.79(0.18)$. Cola $0.15(0)$. Diámetro de huevos 0.06 (0.006) basado en 40.

\section{Discusión}

Cabe destacar que debido a que $C$. acutus es una especie protegida por normas y leyes mexicanas, se aplicó el método mencionado en párrafos anteriores para la obtención de los nemátodos. Las características morfológicas que permitieron la identificación de $D$. helicina en este estudio fueron la longitud de las espículas y la forma del gubernáculo en los machos. Baylis (1947) revisó la taxonomía de las especies Dujardinascaris dujardini (Travassos, 1920) de C. niloticus y D. helicina de $C$. acutus, anteriormente consideradas sinonimias (Baylis, 1923; Gedoelst, 1916), y concluyó que ambas especies son diferentes entre sí en su distribución geográfica y hospederos (D. helicina de cocodrilos americanos, D. dujardini de cocodrilos africanos). Posteriormente, Sprent (1977) las diferenció por la forma del gubernáculo y por la presencia de la papila vulvar.

El ciclo de vida de $D$. helicina es desconocido, pero probablemente involucra un hospedero intermediario acuático que alberga la larva del tercer estadio (L3), ya que la L4 y los adultos se encuentran fijados mediante sus extremos anteriores a la mucosa del estómago de los cocodrilos (Goldberg et al., 1991). En este estudio se observaron crustáceos, peces, aves y reptiles como ítems de los contenidos gástricos de los reptiles analizados, siendo mayoría los peces y crustáceos. De esta maner, podría relacionarse al nemátodo con el tipo de alimento del cocodrilo y definir el o los hospederos potenciales intermediarios para dilucidar el ciclo de vida de esta especie de parásito. De los contenidos gástricos examinados, los que presentaron menores indicadores de infección parasitaria fueron los que pertenecían a D. helicina en cautiverio; esto podría deberse a que a esos ejemplares suele aplicárseles antihelmínticos, y además el alimento suministrado está compuesto por una dieta de carne de res, pollo y ocasionalmente pescado, diferente de la que pudiesen tener en vida libre.

\section{Agradecimientos}

Al personal de la Dirección General de Vida Silvestre, por permitirnos la realización del muestreo mediante el permiso SGPA/DGVS/03993/11. Al personal de la UMA Reptilario
Cipactli de la Universidad de Guadalajara, al personal del ANP Estero El Salado, al Dr. Rafael Lamothe-Argumedo, M. en C. Luis García Prieto y M. en C. David Osorio Sarabia de la Colección Nacional de Helmintos del Instituto de Biología de la UNAM por su guía y apoyo logístico, equipo e instalaciones.

\section{Referencias}

Álvarez-del Toro, M. (1974). Los Crocodylia de México. México, D.F.: Instituto Mexicano de Recursos Naturales Renovables, Conacyt y WWF.

Ayarzagüena, J. (1983). Ecología del caimán de anteojos (Caiman crocodilus) en los llanos de Apure Venezuela. Doñana Acta Vertebrata número especial, 10, 1-136.

Baker, M. R. (1987). Synopsis of the Nematoda parasitic in amphibians and reptiles. Memorial University of Newfoundland. Occasional Papers in Biology, $11,1-325$.

Baylis, H. A. (1923). On the genus Dujardinia gedoelst, with a description of a new genus of Anisakinae from a crocodile. Parasitology, 15, 223-232.

Baylis, H. A. (1947). The nematode genus Dujardinascaris (nom. Nov. pro Dujardina) in Crocodilia, whith a description of a new species. Annals of Natural History, 110, 123-134.

Brooks, D. R. y O'Grady, R. T. (1989). Crocodilian and their helminth parasites: macroevolutionary considerations. American Zoologist, 29, 873-883.

Bush, A. O., Lafferaty, K. D., Lotz, J. M. y Shostak, A. W. (1997). Parasitology meets ecology on its own terms: Margolis et al. revisited. Journal of Parasitology, 83, 575-583.

CITES (Convención sobre el Comercio Internacional de Especies Amenazadas de Fauna y Flora Silvestres). (2007). Apéndices I, II y III. CITES. 23-28 pp. Recuperado el 14 enero 2014, de: http://www.cites.org/esp/app/SSep13.pdf.

Gedoelst, L. (1916). Notes sur la faune parasitaire du Congo Belge. Revue Zoologique Africaine (Bruxelles), 5, 1-90.

Goldberg, S. R., Bursey, C. R. y Aquino-Shuster, A. L. (1991). Gastric nematodes of the Paraguayan Caiman, Caiman yacare (Alligatoridae). Journal of Parasitology, 77, 1009-1011.

Groschaft, J. y Baruš, V. (1970). Studies on the helminth fauna of crocodiles in Cuba. Acta Societatis Zoologicae Bohemoslovae, 34, 289-303.

Heimlich, H. J. (1975). A life-saving maneuver to prevent food-choking. Journal of American Medicine Association, 23, 398-401.

Molin, R. (1860). Trenta specie di Nematoidi. Sitzungsberichte der Kaiserlichen Akademie der Wissenschaften in Wien, Mathematisch-Naturwissenschaftliche Classe, 40, 331-358.

Moravec, F. (2001). Some helminth parasites from Morelet's Crocodile, Crocodylus moreletii, from Yucatán, Mexico. Folia Parasitologica, 48, 47-62.

Moravec, F. y Vargas-Vázquez, J. (1998). First description of the male and redescription of the female of Paratrichosoma recurvum (Nematoda: Capillariidae), a skin-invading parasite of crocodiles in Mexico. Parasitology Research, 84, 499-504.

Peláez, D. y Cruz, F. (1953). Consideraciones sobre el género Acanthostomum Looss, 1899 (Trematoda: Acanthostomidae), con descripción de dos especies de México. Memorias del Congreso Científico en México, 7, 269-284.

Sprent, J. F. A. (1977). Ascaridoid nematodes of amphibians and reptiles: Dujardinascaris. Journal of Helmintology, 51, 251-285. 
Sprent, J. F. A., McKeown, E. A. y Cremin, M. (1998). Dujardinascaris spp. (Nematoda: Ascaridoidea) in Old World crocodilians. Systematic Parasito$\log y, 39,209-222$.

Thorbjarnarson, J., \& Special publication of the Crocodile Specialist Group. (1989). Ecology of the American crocodile Crocodylus acutus. Crocodiles, their ecology, management and conservation. Gland, Switzerland: IUCN Publications, N.S.
Travassos, L. (1920). Contribuçao para a sistematica dos Dicrocoelinae Loos, 1899. Archivos de Escola Superior de Agricultura e Medicina Veterinaria, 3, 7-24.

Villegas, A. y González-Solís, D. (2009). Gastrointestinal helminth parasites of the American Crocodile (Crocodylus acutus) in southern Quintana Roo, México. Herpetological Conservation and Biology, 4, 346-351. 